\section{Successful treatment of immune reconstitution inflammatory syndrome-relat- ed hemophagocytic syndrome in an HIV patient with primary effusion lymphoma}

\author{
Markela-P. Zorzou, ${ }^{1}$ Maria Chini, ${ }^{1}$ \\ Athina Lioni, ${ }^{1}$ Georgios Tsekes, ${ }^{1}$ \\ Thomas Nitsotolis, ${ }^{1}$ Ioannis Tierris, ${ }^{2}$ \\ Nicolaos Panagiotou, ${ }^{3}$ \\ Dimitra Rontogianni, ${ }^{4}$ \\ Nicolaos Harhalakis, ${ }^{5}$ Marios Lazanas ${ }^{1}$ \\ ${ }^{1}$ Infectious Diseases Unit, ${ }^{2}$ Surgery \\ Department, ${ }^{3}$ Hematology Department, \\ Korgialeneio-Benakeio General Hospital \\ of Athens; ${ }^{4}$ Pathology Department, \\ ${ }^{5}$ Hematology and Lymphoma Unit, \\ Evangelismos General Hospital of Athens, \\ Greece
}

\section{Abstract}

Although the connection of [secondary hemophagocytic syndrome (sHS)] with HIV has been well documented, optimal treatment regimen is not well established. This is due not only to the rarity of the syndrome, but also to the heterogeneity of the involved population. Most cases are related to opportunistic infections or malignancies in advanced stage, but many cases are also related to seroconversion, in the primary infection setting. Moreover, in the [antiretroviral treatment (ART)] era, rare cases of ART-related sHS have been reported. In these, often fatal cases, an [immune reconstitution inflammatory syndrome (IRIS)] process is involved, posing a serious challenge to the treating physician. We hereby report a case of successful treatment of an HIV patient with primary effusion lymphoma who experienced sHS shortly after ART onset. Our patient, treated with high dose dexamethasone and gamma globulin, achieved complete remission. This case might hint possible therapeutic insights in the treatment of IRIS-related sHS.

\section{Introduction}

Since the first reports of secondary hemophagocytic syndrome in HIV patients, ${ }^{1}$ much light has been shed to the triggering of hemophagocytic syndrome by the HIV virus. Three distinct patterns of sHS in HIV patients can be described. The most common pattern is seen in the setting of severe immunodeficiency, where HIV has been implicated in secondary HS, either alone, or in concert with a large variety of opportunistic infections and malignancies. ${ }^{2,3}$ However, many cases of sHS have also been described in the primary infection setting, during seroconversion. ${ }^{4} \mathrm{~A}$ third pattern of sHS has emerged in the era of [highly active antiretroviral therapy (HAART)], where very sparse reports connect hemophagocytic syndrome with the onset of HAART, through [immune reconstitution inflammatory syndrome (IRIS)]. In the literature, there are only four reports of IRIS-related sHS, most of them with fatal outcome. ${ }^{5-8}$

\section{Case Report}

A 40-year old male patient presented to the Emergency Department of our hospital complaining of malaise, fatigue, night sweats and fever during the last month. The patient had been tested positive for HIV 7 years ago and had been followed on a regular basis in our Infectious Diseases Unit, until two years ago, when he had insistently denied initiation of HAART and was lost to follow up.

On admission, the patient was in a good clinical condition. Clinical examination was remarkable for a mild splenomegaly and a small focus of Kaposi sarcoma in the ankle. Apart from moderate normocytic anemia and elevated ESR, other blood tests and biochemistry panel were normal (Table 1). His CD4 cell count was 17 cells/uL and HIV viral load 105,000 copies/mL.

Blood cultures for common bacteria and mycobacteria were negative. Serum Cryptococcus neoformans antigen and Wright reaction were negative. PCR amplification for CMV genome was undetectable, while for EBV genome $3.4 \times 10^{3}$ copies $/ \mathrm{mL}$ were detected. Bone marrow aspiration and biopsy were performed and cultures were drawn. PCR amplification for Leishmania infantum was positive in the bone marrow and the serum. However, Giemsa stain of the bone marrow was negative for the parasite and antibodies against K39 antigen in the serum were also negative. In view of the above findings, HAART with abacavir, lamivudine and dolutegravir was initiated in the $6^{\text {th }}$ hospital day (day 0 from that point) and the patient was subsequently treated with liposomal amphotericin, while continuing the diagnostic workup.

Whole body CT scan performed early in the course of the fever had revealed mild lymphadenopathy in the mesenteric region, as well as mild splenomegaly. A PET-18-FDG scan had then been performed, which had raised suspicion of lymphoma in the above lymph nodes. In the meantime, there was a transient amelioration of the patient's fever spikes and
Correspondence: Markela Pagonitsa Zorzou, Patelida str 11, Chios, 82132, Greece.

Tel.: +30.694.4161600

E-mail: mpzorzou@gmail.com

Key words: Hemophagocytic syndrome; Immune reconstitution inflammatory syndrome; IRIS, HIV, ART.

Contributions: the authors contributed equally.

Conflict of interest: the authors declare no potential conflict of interest.

Received for publication: 5 May 2016.

Revision received: 8 November 2016.

Accepted for publication: 10 November 2016.

This work is licensed under a Creative Commons Attribution-NonCommercial 4.0 International License (CC BY-NC 4.0).

(C) Copyright M.-P. Zorzou et al., 2016

Licensee PAGEPress, Italy

Hematology Reports 2016; 8:6581

doi:10.4081/hr.2016.6581

transfusion needs and laparoscopic biopsy of the lymph nodes was postponed.

On day 23 from the initiation of HAART and after 5 days of apyrexia, fever reappeared. The patient reported in good clinical condition. A complete workup with hematology and biochemistry panel, as well as blood cultures, was again unremarkable except for the moderate anemia and the elevated infection markers. CD4 cell count was 39 cells/uL and HIV viral load 51.7 copies/mL. A new CT scan was then performed, which revealed generalized lymphadenopathy, more distinct in the mesenteric area, together with an increase in the spleen size at about $22 \mathrm{~cm}$ maximum diameter, as compared to the previous CT scan. In view of the above findings, IRIS syndrome was suspected and NSAIDs were administered.

While laparascopic biopsy was on schedule, on day 35 , there was an abrupt deterioration of the patient's clinical picture with high fevers, together with profound anemia and thrombopenia. An intensive infectious screening was again nonconclusive. PCR amplification for CMV, HHV-8 and parvoB19 genomes were negative, while PCR amplification for EBV was $1.1 \times 10^{3}$. By that time, there was a remarkable increase of the ferritin level at $6500 \mathrm{mg} / \mathrm{dL}$, which raised the suspicion of hemophagocytic syndrome. Bone marrow aspiration was reperformed, and all three samples (the last one, but also the two previous drawn during this hospitalization) were reviewed separately by two different hematologists under this perspective. In all three samples of bone marrow, neither hemophagocytes, nor any evidence of malignancy could be detected. Based on HLH- 
2004 diagnostic guidelines, the patient had fever, splenomegaly, hemoglogin $<9 \mathrm{~g} / \mathrm{dL}$, platelets $<100 \times 10^{9} / \mathrm{L}$, fasting triglycerides $>265 \mathrm{mg} / \mathrm{dL}$, fibrinogen $<1.5 \mathrm{~g} / \mathrm{L}$ and ferritin $>500$ microgram $/ \mathrm{L}$ and thus fulfilled 5 out of 8 criteria for hemophagocytic syndrome. Nor NK-cell activity nor soluble CD25 could be measured. When HScore was applied, our patient had a score of 222 points, which is translated to a $96 \%$ probability of experiencing reactive hemophagocytic syndrome.

Taking into account the rapid decline of the hemoglobulin and the platelets, salvage treatment with $24 \mathrm{mg}$ dexamethasone divided in 3 doses, together with a 5-day schedule of gamma globulin of $400 \mathrm{mg} / \mathrm{kg}$ of body weight was initiated. Patient's response was impres- sive. Fever vanished in the first 48 hours and all cell lineages gradually recovered within 72 hours. Lymph node biopsy was finally performed on day 45 . In the meanwhile, the patient had fully recovered and on day 50 he was discharged home with a tapering dose of methylprednisone, pending biopsy results. Lymph node biopsy revealed a solid-type primary effusion lymphoma, together with infiltration of the lymph node with Kaposi sarcoma. Both types of lesions abundantly expressed HHV-8 genome. The patient is currently afebrile, on HAART and methylprednisone, in good clinical status, on hematology consultation.

\section{Discussion}

Rather than a distinct disease, hemophagocytic syndrome is increasingly recognized as a clinical syndrome with two main characteristics: the dysregulation of NK and T cells and the cytokine storm resulting in the hyperactivation of macrophages ${ }^{9} \mathrm{An}$ increasing number of inherited or acquired defects in NK and cytotoxic lymphocytes has been implicated in the pathogenesis of hemophagocytic syndrome. Several gene mutations and a number of immune deficiency syndromes have been involved in the familial form. Moreover, even more subtle genetic polymorphisms could be involved, under certain conditions, in the sec-

Table 1. Results of blood tests and biochemistry panel.

\begin{tabular}{|c|c|c|c|c|c|c|}
\hline HAART & 3.11 & 9.11 (HAART DAY 1) & 20.11 & 30.11 & 15.12 & 24.12 \\
\hline Hematocrit (\%) & 25.3 & 22.5 & 26.6 & 24.2 & 15.8 & 30.0 \\
\hline Hemoglobulin (g/dLl) & 8.4 & 7.5 & 8.9 & 8.1 & 5.5 & 9.8 \\
\hline WBC (10/L) & 4300 & 4500 & 4100 & 3800 & 4200 & 4900 \\
\hline Polymorphonuclear (\%) & 51 & 62 & 48 & 36 & 49 & 59 \\
\hline Lymphocytes (\%) & 29 & 20 & 15 & 26 & 39 & 26 \\
\hline Platelets (1000) & 164 & 141 & 80 & 130 & 42 & 317 \\
\hline ESR (mm/lst hr) & 140 & & 57 & & 87 & 13 \\
\hline Glucose (mg/dL) & 98 & 105 & 106 & 117 & 108 & 96 \\
\hline BUN (mg/dL) & 14 & 17 & 18 & 19 & 23 & 24 \\
\hline Creatinine $(\mathrm{mg} / \mathrm{dL})$ & 1.1 & 1.3 & 1.2 & 1.3 & 1.4 & 0.9 \\
\hline Sodium $(\mathrm{mmol} / \mathrm{L})$ & 138 & 133 & 134 & 132 & 134 & 137 \\
\hline Potassium $(\mathrm{mmol} / \mathrm{L})$ & 4.3 & 4.7 & 4.6 & 4.2 & 3.7 & 4.7 \\
\hline Calcium (mg/dL) & 8.7 & 7.8 & 7.4 & & 7.2 & \\
\hline Bilirubin (mg/dL) & 0.55 & 0.51 & 0.26 & & 0.44 & \\
\hline AST (U/L) & 9 & ) & 8 & 16 & 8 & 9 \\
\hline ALT (U/L) & 22 & 33 & 24 & 52 & 22 & 46 \\
\hline GT (U/L) & 33 & 57 & 181 & 62 & 107 & 129 \\
\hline CPK (U/L) & 44 & 22 & 23 & & 27 & 8 \\
\hline $\operatorname{ALP}(\mathrm{U} / \mathrm{L})$ & & 64 & 162 & 79 & 202 & 103 \\
\hline LDH (U/L) & 212 & 168 & 154 & 182 & 184 & 157 \\
\hline Cholesterol (mg\%) & 125 & & & & 210 & 262 \\
\hline $\mathrm{HDL}(\mathrm{mg} / \mathrm{dL})$ & & & & & 9 & 25 \\
\hline $\mathrm{LDL}(\mathrm{mg} / \mathrm{dL})$ & & & & & & 133 \\
\hline Triglycerides (mg/dL) & 287 & & & & 734 & 535 \\
\hline Uric Acid (mg/dL) & 6.1 & 5.0 & 4.8 & & & \\
\hline Total Protein (gr/L) & 69 & 60 & 52 & 57 & & 58 \\
\hline Albumin $(\mathrm{gr} / \mathrm{L})$ & 36 & 28 & 22 & 28 & & 26 \\
\hline Globulin (gr/L) & 33 & 31 & 29 & 29 & & 33 \\
\hline CRP & 59 & 61 & 41 & 10 & & 0.1 \\
\hline Ferritin & 1400 & & & & 6500 & \\
\hline INR & 1.1 & & 1.1 & & & 1.1 \\
\hline APTT (sec) & 32 & & 23 & & & 28 \\
\hline Fibrinogen (mg/dL) & 704 & & 350 & & 445 & \\
\hline HIV RNA (copies/mL) & 105000 & & & & 51 & \\
\hline CD4 (cells/Ul) & 17 & & & & 39 & \\
\hline
\end{tabular}


ondary form. Despite the diversity of the initial defect, all patients with hemophagocytic syndrome share a common final pathway: defective apoptosis and decreased cytotoxicity of NK and $T$ cells, accumulation of the above cells and activation of the cascade, prolonged antigen presentation to $\mathrm{CD} 8 \mathrm{~T}$ lymphocytes, subsequent CD8 excessive activation, impaired downregulation and containment of immune response, excessive activation of $\mathrm{T}$ cells, increased cytokine release and proliferation of macrophages, resulting in erythrocyte, white cell and platelet phagocytosis within the reticuloendothelial system.

HIV alone has been implicated in hemophagocytosis, either in the primary infection setting, ${ }^{4}$ or in advanced disease. ${ }^{10}$ The hypercytokinaemia state, which is well recognized in the HIV setting, enhanced by malignancy, opportunistic infections, or even the initiation of ART, together with virus-related defects in NK-cells and T-cells cytotoxicity, provide the necessary background for a second trigger to fuel the cascade. This double-hit theory could explain the increased incidence of hemophagocytosis in HIV patients. Indeed, in an autopsy study of 56 AIDS patients, histopathological evidence of hemophagocytosis was reported in $20 \%{ }^{11}$

A long list of opportunistic infections or malignancies seems to superimpose to HIV virus resulting in secondary HS. Herpes viruses represent the most common triggering factor. ${ }^{10}$ Apart from EBV, which is directly implicated in the pathogenesis of both familial and secondary form, HHV-8, and CMV have also been implicated in the pathogenesis of hemophagocytosis..$^{3,12-15}$ In the case of HHV-8, this is probably due to the encoding of a functionally active IL-6 like viral protein, which enhances the hemophagocytosis cascade. ${ }^{14}$ Nevertheless, in HHV-8 related hemophagocytosis, a high replication status of the virus has been documented in the patients' peripheral blood. ${ }^{3,10,14,16}$ Such HHV-8 viremia was not evident in our case, not even a low-grade one. Moreover, CMV plasma levels were also negative and EBV levels decreased during the episode of hemophagocytosis. Whether these negative PCR results reflect the low sensitivity of the method used, or are the precocious result of ART initiation, remains unclear. However, this is not conflicting with the final diagnosis of our patient, since high- grade HHV-8 viremia carries an increased risk for the development of Kaposi and almost always accompanies the exacerbations of multicentric Castleman disease, but, on the other hand, PCR positivity for HHV-8 is not a prerequisite for the above entities and its use for diagnostic reasons is currently discouraged.

Lymphohyperplastic diseases, have also been implicated in the pathogenesis of secondary hemophagocytosis in HIV patients. ${ }^{2,3,10}$
Indeed, in the HAART era, lymphomas account for the majority of reactive hemophagocytosis in the largest series published, ${ }^{3}$ probably due to the increased survival and the decrease of opportunistic infections. Our patient was finally diagnosed with an HIV-related solid-type primary effusion lymphoma. While this could represent a potential triggering factor, two facts argue against this hypothesis: despite lymphoma, our patient was clinically stable till the initiation of HAART. Moreover, there were signs of complete remission in the biopsy specimen, which probably reflects the beneficial effect of HAART.

The correlation of HAART with hemophagocytosis has been proposed in four case reports in the last decade. ${ }^{5-8} \mathrm{~A}$ review of these reports draws a few important hints: Three of the five patients reported, were finally diagnosed with an underlying lymphohyperplastic disease. When reported in the paper, a very low CD4 count was present prior to the initiation of HAART. Even though we ignore the possible significance of these findings, our patient shared both these features.

The onset of HAART results in a reduction in HIV viral load and an increase in CD4 cell count. This immune restoration rarely results in a paradoxical inflammatory response to subclinical infectious or noninfectious diseases termed inflammatory reconstitution immune syndrome. IRIS represents a disproportionate inflammatory response to various antigens, resulting in a cytokine storm. In clinical terms, IRIS is a diagnosis of exclusion, closely related to the initiation of HAART, with clinical manifestations those of the underlying entity. Such unmasking of the underlying disease could trigger the hemophagocytosis cascade, which would otherwise remain silent. Our patient had a transient amelioration of his symptoms, till one month after the beginning of HAART, where fever gradually reappeared. He also manifested an impressive decrease in his viral load, which is compatible with IRIS syndrome. Moreover, no other culprit could be found, despite an extensive workup. It is worth mentioning that at the time IRIS was suspected, the patient did not fulfill criteria for hemophagocytosis.

In a review of the five cases of IRIS-related hemophagocytosis reported, a fatal outcome was the rule in all but one patient. This could probably reflect not only the difficulty in diagnosis, but also the complexity of treatment in HAART related cases. Indeed, optimal treatment of secondary HS in HIV patients is still ambiguous. A bundle of interventions has been proposed in the literature. Supportive care is the cornerstone of treatment. Pathogen directed therapy has been proposed, in infectionrelated cases. Withdrawal of the potential trigger seems important, in non-infectious cases. HLH 2004 protocol has been addressed to HIV patients with controversial results. HAART has been adopted in HS in the setting of severe immunodeficiency.

Immunomodulatory interventions have also been proposed, in an attempt to reverse the cytokine cascade. ${ }^{16}$ High doses of corticosteroids seem to have a role in the treatment of secondary hemophagocytosis, even though there are sparse data in the HIV positive population. Etoposide seems effective, especially in EBV-associated syndrome. ${ }^{16}$ Therapeutic immunomodulatory agents, particularly infliximab has been proposed in an attempt to attenuate the cytokine storm. Even more recently, the use of tocilizumab, a humanized monoclonal antibody against IL-6 receptor, has emerged as an appealing agent in cases of refractory hemophagocytic syndrome, in patients with autoimmune disorders and a clinical trial for its use in the pediatric population is currently ongoing. ${ }^{17,18}$ In the same context, splenectomy has also been proposed in a few case reports, where disease bulk was confined to the spleen.

Intravenous immunoglobulin has also been proposed in the treatment of hemophagocytic syndrome though, to our knowledge, only in the HIV negative setting. In one study of 17 patients with sHS, $59 \%$ response rate was noted. ${ }^{19}$ The response was more pronounced in the infection-related group. Cyclosporine has also been used in lymphoma associated hemophagocytosis where IVIG yielded poor results.

Reactive hemophagocytic syndrome in the HIV setting carries a dismal prognosis, and this was especially true in the pre-ART era. In the few case reports in the literature, where IRIS is related to hemophagocytic syndrome, the outcome was rather unfavorable in most of the cases. The favorable outcome of our patient, could, thus, either represent a milder form of the syndrome, a more favorable underlying malignancy, or be the result of a successful treatment approach. Indeed, hemophagocytic syndrome and IRIS syndrome seem to share common pathogenetic mechanisms and probably reflect different magnitude of the hyperinflammation process. Under this prespective, the favorable outcome of our patient could be attributed to the milder form of IRISrelated HS, versus other forms of hemophagocytic syndrome. Further reports will likely elucidate the benign nature of IRIS-related HS.

However, as mentioned above, this syndrome was lethal in most of the cases reported in the literature. When our patient is compared to these cases, three more parameters could be implied: the impact of the underlying malignancy, the continuation of ART despite IRIS syndrome and the treatment with IVIG. Indeed, the continuation of HAART is increasingly proposed in IRIS syndrome in the literature. Even though, in our case, IRIS resulted in a dreadful complication, such an approach had 
multiple advantages. Taking into account the complete remission of the underlying lymphoma, longer follow up is necessary to estimate the durability of this result.

On the other hand, what is even more promising, is, to our opinion, the effect of IVIG in the reversal of hemophagocytosis and cytokine cascade. This is the first report of IVIG administration in HAART-related hemophagocytosis if not in HIV-related hemophagocytosis. Such an approach, even in the context of one case report, merits special attention, given the poor prognosis of hemophagocytosis in this patients' population.

\section{Conclusions}

IRIS-related hemophagocytic syndrome is a rare but existing entity, with only four reports in the literature, most of them with fatal outcome. Most of these patients had an underlying lymphoma. This is also the case in our patient, where HAART continuation and the combination of IVIG and dexamethasone proved life-saving. Our experience could hint possible therapeutic insights to the treatment of this dreadful entity, even though its rarity deters us from drawing safer conclusions.

\section{References}

1. Bourquelot $\mathrm{P}$, Oksenhendler E, Wolff M, et al. [Hemophagocytic syndrome in HIV infection] Presse Med 1993;11:1217-20. [Article in French].

2. Grateau G, Bachmeyer C, Blanche P, et al. Haemophagocytic syndrome in patients infected with the human immunodeficiency virus: nine cases and a review. J Infect 1997;34:219-25.

3. Fardet L, Lambotte 0 , Meynard J, et al.
Reactive haemophagocytic syndrome in 58 HIV-1-infected patients: clinical features, underlying diseases and prognosis. AIDS 2010;24:1299-306.

4. Chen TL, Wong WW, Chiou TJ. Hemophagocytic syndrome: an unusual manifestation of acute human immunodeficiency virus infection. Int $\mathrm{J}$ Hematol 2003;78:450-2.

5. Huang D, Wu J, Hamill R. Reactive hemophagocytosis associated with the initiation of highly active antiretroviral therapy (HAART) in a patient with AIDS. Scand J Infect Dis 2004;36:516-9.

6. Cuttelod M, Pascual A, Baur Chaubert A, et al. Hemophagocytic syndrome after highly active antiretroviral therapy initiation: a life-threatening event related to immune restoration inflammatory syndrome? AIDS 2008;22:549-51.

7. Kanitez M., Kapmaz M, Alpay N, et al. hemophagocytic syndrome associated with immune reconstitution inflammatory syndrome in a patient with AIDS related Burkitt's leukemia/lymphoma. Case Rep Med 2014;2014:1-3.

8. Safdar S, Rehman J, Samman E, Bahabri N. Fatal hemophagocytic syndrome as a manifestation of immune reconstitution syndrome in a patient with acquired immunodeficiency syndrome. Saudi Med J 2013;34:861-4.

9. Lazanas M, Perronne C, Lomverdos D, et al. [Visceral leishmaniasis disclosed by histiocytosis with erythrophagocytosis. Presse Med 1990;21:765. [Article in French].

10. Doyle T, Bhagani S, Cwynarski K. Haemophagocytic syndrome and HIV. Curr Opin Infect Dis 2009;22:1-6.

11. Niedt GW, Schinella RA. Acquired immunodeficiency syndrome. Clinicopathological study of 56 autopsies. Arch Pathol Lab Med 1985; 109:727-34.

12. Khagi S, Danilova O, Rauwerdink C.
Hemophagocytic syndrome in a patient with human immunodeficiency virus, Epstein Barr viremia and newly diagnosed Hodgkin lymphoma. Hematol Oncol Clin Case Stud 2012;10:260-5.

13. Kim SB, Shrivastava MS, Strakhan M. A rare cause of acquired immune deficiency syndrome related pancytopenia. Hematol Rep 2015;9:5475.

14. Fardet L, Blum L, Kerob D, et al. Human Herpes Virus 8-associated hemophagocytic lymphohistiocytosis in human immunodeficiency virus-infected patients. Clin Infect Dis 2003;37:285-91.

15. Rouphael N, Talati N, Vaughan C, et al. Infections associated with haemophagocytic syndrome. Lancet Infect Dis 2007;7: 814-22.

16. Stebbing J, Ngan S, Ibrahim H, et al. The successful treatment of haemophagocytic syndrome in patients with human immunodeficiency virus-associated multi-centric Castleman's disease. Clin Exp Immunol 2008;154:399-405.

17. Rios-Fernández R, Callejas-Rubio JL, García-Rodríguez S, et al. tocilizumab as an adjuvant therapy for hemophagocytic lymphohistiocytosis associated with visceral leishmaniasis. Am J Ther 2016;23: e1193-6.

18. Faguer S, Vergez F, Peres $M$, et al. Tocilizumab added to conventional therapy reverses both the cytokine profile and CD8+Granzyme+ T-cells/NK cells expansion in refractory hemophagocytic lymphohistiocytosis. Hematol Oncol 2016;34:557.

19. Larroche C, Bruneel F, Andre M, et al. [Intravenously administered gamma-globulins in reactive hemophagocytic syndrome. Multicenter study to assess their importance, by the immunoglobulins group of experts of CEDIT of the AP-HP]. Ann Med Interne (Paris) 2000;151:533-9. [Article in French]. 